\title{
Cross-sectional and longitudinal associations of neighborhood characteristics with inflammatory markers: Findings from the multi-ethnic study of atherosclerosis ${ }^{2}$, 访婂
}

\author{
Aydin Nazmi ${ }^{\mathrm{a}, *}$, Ana Diez Roux ${ }^{\mathrm{a}}$, Nalini Ranjit ${ }^{\mathrm{a}}$, Teresa E Seeman ${ }^{\mathrm{b}}$, Nancy S. Jenny ${ }^{\mathrm{c}}$ \\ a Department of Epidemiology, 109 Observatory Street, SPH Tower, University of Michigan School of Public Health, Ann Arbor, MI 48109, USA \\ b Department of Medicine, Division of Geriatrics, University of California, Los Angeles, 10945 Le Conte Avenue, Suite 2339, Los Angeles, CA 90095, USA \\ ${ }^{\mathrm{c}}$ Department of Pathology, University of Vermont College of Medicine, Burlington, VT 05405, USA
}

\section{A R T I C L E I N F O}

\section{Article history:}

Received 23 December 2009

Received in revised form

7 July 2010

Accepted 12 July 2010

\section{Keywords:}

Inflammation

Fibrinogen

Interleukin-6

C-reactive protein

Neighborhood

\begin{abstract}
A B S T R A C T
We investigated cross-sectional associations of neighborhood deprivation, problems, safety, and cohesion with circulating levels of fibrinogen, interleukin-6, and C-reactive protein $(n=5370)$ and longitudinal associations with changes in IL-6 over a 3-4 year period $(n=946)$. In cross-sectional analyses, higher levels of neighborhood deprivation and problems were associated with higher levels of all three inflammatory markers, whereas higher levels of safety were associated with lower levels. Fibrinogen remained associated with all neighborhood characteristics except cohesion and IL-6 remained associated with safety after adjustment for race and SES. In longitudinal analyses, higher levels of neighborhood deprivation and problems, and lower levels of safety were associated with greater longitudinal increases in IL-6 after adjustment for age, sex, race, and SES. These findings were not substantially modified by further risk factor adjustment. Although findings regarding different inflammatory markers were mixed, the longitudinal results that are less limited by race confounding suggest that inflammatory pathways may contribute to neighborhood differences in cardiovascular disease risk.
\end{abstract}

(c) 2010 Elsevier Ltd. All rights reserved.

\section{Introduction}

A number of studies have documented associations of neighborhood characteristics with cardiovascular outcomes that persist after accounting for individual-level measures of socioeconomic position (Cubbin and Winkleby, 2005; Leclere et al., 1998; Sundquist et al., 2004; Diez Roux et al., 2001, 1995; Franzini and Spears, 2003; Chaix et al., 2007; Winkleby et al., 2007). However, considerable debate exists on whether these associations are causal and the mechanisms mediating these associations have not been fully identified. In some cases, associations of neighborhood characteristics with cardiovascular outcomes appear to persist after additional control for established cardiovas-

This manuscript was presented as an abstract at the 42nd Annual Society for Epidemiologic Research Meeting in Anaheim, CA in June 2009.

Funded by NIH.

* Corresponding author. Present address: Department of Food Science and Nutrition, 1 Grand Avenue, California Polytechnic State University, San Luis Obispo, CA, USA. Tel.: +1 805756 6183; fax: +1 8057561146.

E-mail addresses: nazmi@calpoly.edu (A. Nazmi), adiezrou@umich.edu (A. Diez Roux), nranjit@umich.edu (N. Ranjit), tseeman@mednet.ucla.edu (T.E. Seeman), nancy.jenny@uvm.edu (N.S. Jenny). cular risk factors, suggesting that other mediators may be involved (Diez Roux et al., 2001).

Systemic inflammation, as indexed by various circulating markers, has emerged as a possible cardiovascular risk factor (Willerson and Ridker, 2004). The inflammatory markers fibrinogen, interleukin-6 (IL-6), and C-reactive protein (CRP) are known to be inversely associated with individual-level socioeconomic factors (Jousilahti et al., 2003; Wilson et al., 1993; Nazmi and Victora, 2007; Ranjit et al., 2007a; Ramsay et al., 2008) but there are plausible mechanisms through which neighborhood context may affect levels of inflammatory markers independently of individual-level socioeconomic indicators. These include possible effects of neighborhood context on behavioral factors (such as diet, physical activity, or smoking) (Diez Roux and Mair, 2010) linked to inflammation, neighborhood differences in the risk of acquiring infectious diseases linked to inflammatory processes (Acevedo-Garcia, 2000), neighborhood differences in air pollution levels (O'neill et al., 2003) as well as effects of neighborhood environments on stress-related physiological processes hypothesized to play a role in the inflammatory process (Mcdade et al., 2006; Ranjit et al., 2007b).

Very few studies have investigated associations of neighborhood characteristics with inflammatory markers (Pollitt et al., 2007; Petersen et al., 2008) and no studies of which we are aware 
have moved beyond neighborhood socioeconomic characteristics to investigate more specific neighborhood attributes, or have examined associations of neighborhood characteristics with changes in inflammatory markers over time. Finding that neighborhood-level factors are associated with biological precursors of cardiovascular risk would strengthen evidence for causal effects of neighborhoods on the development of cardiovascular disease.

Using data from an ancillary study to the multi-ethnic study of atherosclerosis (MESA), we investigated cross-sectional associations of several neighborhood characteristics (deprivation, safety, cohesion, and problems) with circulating levels of inflammatory markers and longitudinal changes in inflammatory markers in a large population-based multi-ethnic sample with detailed measures of neighborhood characteristics, inflammatory markers, and potential confounders.

\section{Methods}

The multi-ethnic study of atherosclerosis (MESA) is a population-based longitudinal study of the prevalence, correlates, and progression of subclinical cardiovascular disease (CVD). The cohort comprised 6814 individuals aged $45-84$ years with $38 \%$ white, 28\% black, 22\% Hispanic, and 12\% Chinese. Participants were recruited from six US communities using a variety of population-based approaches and were free of clinical CVD at the time of the baseline visit. Detailed information on the study objectives and design has been published elsewhere (Bild et al., 2002). Cross-sectional analyses reported here employed data collected as part of the baseline examination conducted between 2000 and 2002. Longitudinal analyses used follow-up data available 3-4 years after baseline (2004-2006) collected on a subsample of white, black, and Hispanic participants from the New York and Los Angeles sites. The follow-up sample was composed of participants in an ancillary study to MESA, which collected follow-up information on approximately 1000 white, black, and Hispanic participants at two of the MESA sites (Ranjit et al., 2009). Participants were invited to enroll in the ancillary study in the order in which they attended the visit resulting in an approximately random sample (Ranjit et al., 2009).

Three inflammatory markers (fibrinogen, IL-6, and CRP) linked to cardiovascular events in prior work (Pahor et al., 1999; Tracy, 1999) were investigated in cross-sectional analyses. Fibrinogen $(\mathrm{mg} / \mathrm{dL})$ and CRP $(\mathrm{mg} / \mathrm{L})$ were measured using the BNII nephelometer ( $\mathrm{N}$ high sensitivity $\mathrm{CRP}$ and $\mathrm{N}$ antiserum to human fibrinogen, Dade Behring, Deerfield, IL). IL-6 (pg/mL) was measured by ultrasensitive ELISA (R\&D Systems, Minneapolis, $\mathrm{MN})$. Analytic coefficients of variation were $2.6 \%, 6.3 \%$, and $3.6 \%$ for fibrinogen, IL-6, and CRP, respectively. CRP and IL-6 were logtransformed prior to analyses due to skewed distributions. Follow-up measures of IL-6 were available on a subsample of 946 white, black, and Hispanic participants from the New York and Los Angeles MESA sites (mean follow-up time 3.9 years). All assays were performed at the MESA Central Laboratory located at the University of Vermont.

Neighborhood of residence (proxied by census tract) was assigned to each MESA participant based on residential addresses at baseline. Four neighborhood domains were investigated: neighborhood deprivation, safety, cohesion, and problems. Neighborhood deprivation was used as a summary measure of the neighborhood environment. A deprivation score for each neighborhood was created based on a factor analysis of 19 census tractlevel variables reflecting dimensions of race/ethnicity, family structure, housing, residential stability, crowding, education, employment, occupation, and income/wealth available in the
2000 US Census. Six variables, which accounted for $61 \%$ of the variance (percent vacant housing, percent with no telephone, percent with no vehicle, percent unemployed, median household income, and percent poverty) loaded on the first factor were used in these analyses. The deprivation factor was the weighted sum of the six standardized variables (Mujahid et al., 2007). The factor score was converted to $z$ scores.

The three other neighborhood domains investigated - problems, safety, and cohesion - were assessed using questionnaires administered to MESA participants. These three domains were selected for investigation because they capture aspects of neighborhoods that may be related to levels of inflammatory markers through their effects on health behaviors or psychosocial processes related to inflammation. Each domain was assessed using scales linked to cardiovascular-related outcomes in prior work (Mujahid et al., 2007; Auchincloss et al., 2008; Echeverría et al., 2008). Seven questions were used to determine the neighborhood problems score. These items referred to the state of the neighborhood sidewalks, trash and litter in the neighborhood, playgrounds/parks, adequate shopping, heavy traffic/speeding, excessive noise and violence in the neighborhood. For each item participants were asked to indicate whether the issue in question was a problem using a 4 point Likert scale ("often" to "never"). The neighborhood safety score was based on three items (using a 5 point Likert scale: "strongly agree" to "strongly disagree") regarding neighborhood violence, crime, and the ability to walk in the neighborhood without fear. Neighborhood cohesion was scored according to five items (using a 5 point scale as above) on neighbors' willingness to help each other, neighbors getting along, neighbors trusting each other and shared/common values. Likert scales used to collect data on individual items were summed to create composite scores. Higher scores for each characteristic referred to more neighborhood problems, greater safety, and greater social cohesion. The scales had acceptable validity and reliability for the measurement of neighborhoodlevel domains (Mujahid et al., 2007; Echeverria et al., 2004).

Because aggregation across multiple respondents may reduce error arising from individual subjectivities and improve the validity of the measure (Mujahid et al., 2007), the neighborhood characteristics of each MESA participant was estimated by averaging the responses of all other MESA participants living within the same census tract. All neighborhood variable values were converted to $z$ scores for analysis.

Baseline covariates included age, race/ethnicity, socioeconomic status, use of anti-inflammatory medications, recent history of acute infection, physical activity, smoking, alcohol drinking, body mass index (BMI), waist circumference, impaired fasting glucose, and diabetes. All of these variables have been linked to levels of inflammatory markers and could plausibly confound and/or mediate any neighborhood effects on inflammation. Race/ethnicity was self-reported based on questions modeled on the year 2000 census. Education was collected in nine categories (no schooling, 1-8 years, 9-11 years, high school graduate or GED certificate, some college but no degree, technical school certificate, associate's degree, bachelor's degree, graduate/ professional degree) and income in 13 categories (ranging from $\$<5000$ to $100000+$ ). Some of these categories were collapsed for descriptive analyses. Use of hormone replacement therapy, aspirin, oral anti-inflammatory agents, lipid-lowering drugs, and non-steroidal anti-inflammatory drugs during the baseline visit was grouped into a single dichotomous variable (yes to any vs. none). Having had an acute infection (bronchitis, cold/flu, fever, pneumonia, sinusitis, urinary, or tooth infection) in the 2 weeks previous to the baseline interview was coded as positive for recent infection. Physical activity was assessed using a standardized questionnaire (Ainsworth et al., 1999). Level of intentional 
physical activity (walking for exercise, sports, and conditioning activities) was calculated in MET-min/week and investigated as a continuous variable in regression models; for descriptive analyses, the lowest tertile of intentional activity was used to define sedentary behavior. Tobacco smoking and alcohol habits were each categorized as never, current, or ex-user. Dietary assessment at baseline was performed using principal components analysis based on a 120-item food frequency questionnaire and 47 investigator-defined food groups. Four main dietary patterns were identified by factor analysis and named according to the types of foods loading on the highest of each pattern. These groups were fats and processed meats; vegetables and fish; beans, tomatoes and refined grains; whole grains and fruit. Factor scores were calculated for each participant and ranged overall from -1.82 to 12.30 , representing the lowest and highest score for each dietary group, respectively (Nettleton et al., 2006). Weight, height, and waist circumference were measured using standardized procedures as part of the baseline exam. BMI was calculated as weight over height in meters squared. BMI and waist circumference were investigated as continuous variables. Diabetes was defined as self-reported ongoing medical treatment for diabetes or fasting glucose $>125 \mathrm{mg} / \mathrm{dL}$ and impaired fasting glucose was defined as fasting glucose of $100-125 \mathrm{mg} / \mathrm{dL}$ (American Diabetes Association 2003). Updated measures of covariates were also available at the time of the follow-up IL-6 assessment on the subsample. The Institutional Review Board at each participating MESA site reviewed the study and written informed consent was collected from all participants.

\subsection{Statistical methods}

In cross-sectional analyses age-adjusted mean fibrinogen and geometric mean CRP and IL-6 levels by quartiles of neighborhood domains were estimated using linear regression. P-values for trend were obtained by including neighborhood categories as ordinal variables in regression models. Mixed models with random intercept at the census tract level that account for the clustering of individuals within neighborhoods were employed to estimate associations between neighborhood domains and inflammatory markers before and after adjustment for sets of covariates. Five models were fitted: model 1 adjusted for age and sex; model 2 for age, sex, education, and family income; model 3 for age, sex, and race/ethnicity; model 4 for age, sex, race/ ethnicity, education, and family income; model 5 included all of the above plus selected confounders or possible mediators of the association between neighborhood domains and inflammation (BMI, waist circumference, physical activity, dietary pattern score, smoking, alcohol intake, recent acute infection, medication use, diabetes, and impaired fasting glucose). Intraclass correlation coefficients of inflammatory markers within neighborhoods were estimated using variance components of the mixed models.

In longitudinal analyses, mixed models were used to model the mean annual change in IL- 6 between baseline and follow-up as a function of neighborhood domains in the subsample. Mean annual change in IL-6 levels was calculated by subtracting levels of IL-6 at baseline (2000-2002) from levels at follow-up (2004-2006) and dividing by mean follow-up time in years. We fit models analogous to models 1-5 described for cross-sectional analyses. In sensitivity analyses we also examined confounders/ mediators as time-varying covariates both in absolute levels and as change between baseline and follow-up. These results were similar to those of model 5 and are not reported. Baseline IL-6 level was included as a covariate in all models. In sensitivity analyses, the impact of measurement error in the baseline IL-6 measures was investigated using regression calibration (Hardin et al., 2003) with a known error of $6.3 \%$ based on the coefficient of variation for IL-6 measurements. Results were similar and therefore only the simpler models are reported. In both crosssectional and longitudinal analyses we also examined the contribution of neighborhood problems, safety, and cohesion to differences in inflammatory markers by examining the change in the deprivation coefficient when the other neighborhood-level variables were added.

\section{Results}

Of the 6814 MESA participants at baseline, 6599 had complete data on all inflammatory markers of which 5370 had complete data on neighborhood characteristics and were included in the cross-sectional analyses. These participants were distributed in 1245 census tracts with a median (IQR) of 10 (3-25) participants per tract. The mean age of the sample was 62.2 years $( \pm 10.2)$; $39 \%$ were white, $28 \%$ black, $22 \%$ Hispanic, and $12 \%$ Chinese. $35 \%$ had at least a college degree whereas $18 \%$ had not completed high school. 38\% had an annual family income less than $\$ 30,000$ whereas $23 \%$ had more than $\$ 75,000$ of income. Median (IQR) for fibrinogen in men and women, respectively, was $323 \mathrm{mg} / \mathrm{dL}$ (285-369) and $353 \mathrm{mg} / \mathrm{dL}$ (308-404). Median (IQR) for IL-6 and CRP in men and women were $1.13 \mathrm{pg} / \mathrm{mL}(0.73-1.80)$ and $1.27 \mathrm{pg} / \mathrm{mL}(0.82-1.95)$, and $1.43 \mathrm{mg} / \mathrm{L}(0.70-3.15)$ and $2.56 \mathrm{mg} / \mathrm{L}$ (1.05-5.69), respectively.

Table 1 shows selected characteristics of MESA participants by quartile of neighborhood deprivation score at baseline. Individuals living in more deprived neighborhoods were more likely to be black, Hispanic and had lower education and income than those living in less deprived neighborhoods $(p<0.001$ for all). Higher neighborhood deprivation was also associated with less medication use, higher prevalence of recent infections, physical inactivity, more smoking, less alcohol intake, higher BMI and waist circumference and higher prevalence of impaired fasting glucose and diabetes. More deprived neighborhoods had lower safety and cohesion scores and higher neighborhood problems scores $(p<0.001$ for all). Individuals living in more deprived neighborhoods also had higher levels of inflammatory markers $(p<0.001$ for all). Correlations between the four neighborhood level variables ranged from 0.37 for deprivation and cohesion to -0.66 for deprivation and safety $(p<0.001$ for both). The percent of variance in inflammatory markers between neighborhoods (the intraclass correlation coefficient estimated from a model without covariates) ranged from $1.1 \%$ for fibrinogen in men to $8.2 \%$ for IL-6 in women. The between-neighborhood variance differed significantly from zero in all models.

Figs. 1-3 show age-adjusted means for fibrinogen, IL-6, and $\mathrm{CRP}$ at baseline in men and women by quartile of neighborhood characteristics score. In both sexes, there were graded and statistically significant associations between levels of all three inflammatory markers and neighborhood variables. Higher levels of neighborhood deprivation and higher levels of neighborhood problems were associated with higher levels of all three inflammatory markers $(p<0.001$ for all, except CRP levels in women, $p=0.009$ ). Higher levels of neighborhood safety were associated with lower levels of inflammatory markers $(p<0.001$ for all, except CRP level in women, $p=0.01$ ). Results for the neighborhood cohesion variable were less consistent: higher neighborhood social cohesion was associated with lower levels of fibrinogen in men $(p=0.003)$ but with higher levels of CRP in women $(p=0.009)$. Other differences by social cohesion were not statistically significant.

In general, patterns were similar in men and women and there was no consistent evidence of interactions by sex (of 12 
Table 1

Selected characteristics of MESA participants (2000-2002) by quartile of neighborhood deprivation score $(N=5370)$.

\begin{tabular}{|c|c|c|c|c|c|}
\hline & \multicolumn{4}{|c|}{ Quartile of neighborhood deprivation score } & \multirow[t]{2}{*}{$P$ for trend } \\
\hline & I-Least deprived & II & III & IV-Most deprived & \\
\hline Mean (SD) age, years & $62.3(9.9)$ & $61.4(10.0)$ & $61.8(10.3)$ & $62.2(10.4)$ & - \\
\hline$\%$ Female & 48.4 & 52.1 & 54.1 & 55.3 & 0.001 \\
\hline Race/ethnicity (\% distribution) & & & & & $<0.001$ \\
\hline White & 58.0 & 54.1 & 34.4 & 8.8 & \\
\hline Black & 14.4 & 21.3 & 29.4 & 44.2 & \\
\hline Hispanic & 11.9 & 15.7 & 20.7 & 39.2 & \\
\hline Chinese & 15.8 & 9.0 & 15.6 & 7.8 & \\
\hline Education (\% distribution) & & & & & $<0.001$ \\
\hline Not high school graduate & 5.8 & 11.3 & 19.2 & 31.3 & \\
\hline High school graduate & 15.7 & 17.1 & 19.3 & 31.5 & \\
\hline Some college & 29.4 & 29.4 & 29.5 & 26.2 & \\
\hline College graduate & 49.1 & 42.3 & 32.1 & 21.0 & \\
\hline Income (\% distribution) & & & & & $<0.001$ \\
\hline$<16000$ & 8.8 & 12.1 & 20.1 & 30.2 & \\
\hline 16000-29999 & 10.5 & 16.4 & 22.8 & 24.2 & \\
\hline 30000-49999 & 22.6 & 22.8 & 25.2 & 23.3 & \\
\hline $50000-74999$ & 20.2 & 20.0 & 15.1 & 12.7 & \\
\hline $75000+$ & 37.9 & 28.8 & 16.8 & 9.6 & \\
\hline$\%$ Current medication use & 59.6 & 58.2 & 51.4 & 47.2 & $<0.001$ \\
\hline \% Recent infection & 19.9 & 22.3 & 23.1 & 30.4 & $<0.001$ \\
\hline$\%$ Physically inactive ${ }^{a}$ & 26.8 & 30.6 & 36.3 & 37.3 & $<0.001$ \\
\hline \multicolumn{6}{|l|}{ Mean (SD) dietary pattern score ${ }^{\mathrm{b}}$} \\
\hline Fats and processed meats & $0.03(0.95)$ & $0.09(1.0)$ & $0.01(1.04)$ & $-0.14(0.93)$ & $<0.001$ \\
\hline Vegetables and fish & $0.09(1.12)$ & $-0.08(0.94)$ & $0.02(0.95)$ & $-0.02(0.92)$ & 0.0001 \\
\hline Beans, tomatoes, and refined grains & $-0.19(0.69)$ & $-0.05(0.93)$ & $0.06(1.06)$ & $0.16(1.16)$ & $<0.001$ \\
\hline Whole grains and fruits & $0.09(0.93)$ & $0.09(0.99)$ & $-0.04(1.04)$ & $-0.09(1.0)$ & $<0.001$ \\
\hline$\%$ Current smoker & 7.9 & 13.3 & 12.9 & 16.4 & $<0.001$ \\
\hline \% Current alcohol drinker & 63.5 & 60.8 & 52.4 & 48.0 & $<0.001$ \\
\hline Mean (SD) BMI, $\mathrm{kg} / \mathrm{m}^{2}$ & $27.6(5.0)$ & $28.2(5.3)$ & $28.3(5.5)$ & $29.1(5.7)$ & $<0.001$ \\
\hline Mean (SD) waist circumference, $\mathrm{cm}$ & $96.3(13.9)$ & $98.4(14.3)$ & $97.8(14.4)$ & $99.3(14.3)$ & $<0.001$ \\
\hline \% Diabetes ${ }^{c}$ & 8.7 & 10.4 & 12.7 & 15.7 & $<0.001$ \\
\hline$\%$ Impaired glucose tolerance ${ }^{\mathrm{d}}$ & 11.5 & 11.1 & 12.4 & 14.2 & 0.01 \\
\hline \multicolumn{6}{|l|}{ Mean (SD) neighborhood scores ${ }^{\mathrm{e}}$} \\
\hline \multirow[t]{2}{*}{ Safety } & $4.1(0.4)$ & $3.8(0.3)$ & $3.5(0.3)$ & $3.2(0.2)$ & $<0.001$ \\
\hline & IQR: $3.9-4.3$ & IQR: $3.4-4.0$ & IQR: $3.3-3.7$ & IQR: $3.0-3.5$ & \\
\hline \multirow[t]{2}{*}{ Cohesion } & $3.6(0.2)$ & $3.6(0.2)$ & $3.5(0.2)$ & $3.3(0.2)$ & $<0.001$ \\
\hline & IQR: $3.5-3.8$ & IQR: $3.4-3.7$ & IQR: $3.3-3.8$ & IQR: $3.1-3.6$ & \\
\hline \multirow[t]{2}{*}{ Problems } & $1.3(0.2)$ & $1.4(0.2)$ & $1.5(0.2)$ & $1.7(0.2)$ & $<0.001$ \\
\hline & IQR: $1.2-1.4$ & IQR: $1.3-1.6$ & IQR: $1.4-1.6$ & IQR: $1.5-1.9$ & \\
\hline Median (IQR) fibrinogen, mg/dL & $326(285-373)$ & $333(292-382)$ & 339 (298-389) & $351(307-407)$ & $<0.001$ \\
\hline Median (IQR) IL-6, pg/mL & $1.05(0.70-1.74)$ & $1.17(0.76-1.78)$ & $1.23(0.78-1.92)$ & $1.28(0.84-1.95)$ & $<0.001$ \\
\hline Median (IQR) CRP, mg/L & $1.64(0.71-3.94)$ & $1.87(0.84-4.16)$ & $1.86(0.86-4.08)$ & $2.15(0.93-4.75)$ & $<0.001$ \\
\hline$N(\%)$ & $1350(25.1)$ & $1569(29.2)$ & $1186(22.1)$ & $1265(23.6)$ & \\
\hline
\end{tabular}

$P$ values by chi-squared test for trend (log-transformed for IL-6 and CRP) and ANOVA for dietary pattern scores, BMI, waist circumference and neighborhood scores.

a Physical inactivity: lowest tertile of MET-minutes per week of intentional activity.

${ }^{\mathrm{b}}$ Dietary pattern score ranges: fats $-1.49-9.89$, vegetables $-1.42-12.30$, beans $-1.31-11.72$, whole grains $-1.83-7.74$.

c Diabetes: currently under treatment for diabetes or fasting plasma glucose $\geq 126 \mathrm{mg} / \mathrm{dL}$.

d Impaired glucose tolerance: fasting plasma glucose $100-125 \mathrm{mg} / \mathrm{dL}$.

e Neighborhood score ranges: safety $1.3-5.0$, cohesion $2.4-4.6$, problems $1.0-2.6$.

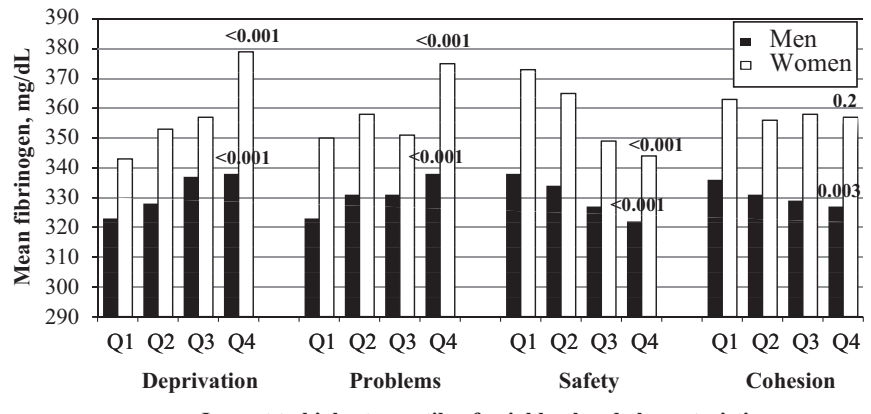

Lowest to highest quartile of neighborhood characteristic

Fig. 1. Mean fibrinogen levels $(\mathrm{mg} / \mathrm{dL})$ per quartile of neighborhood characteristic according to sex among MESA participants $(N=5370)$. Age-adjusted $P$ values for trend across quartiles are shown at the highest quartile.

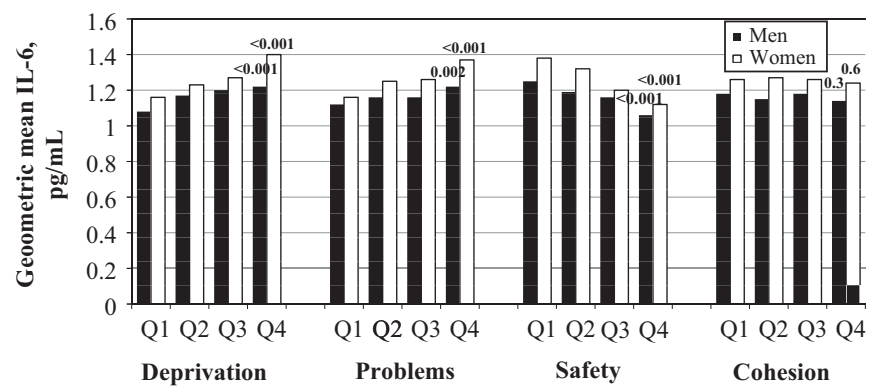

Lowest to highest quartile of neighborhood characteristic

Fig. 2. Geometric mean interleukin-6 levels $(\mathrm{pg} / \mathrm{mL})$ per quartile of neighborhood characteristic according to sex among MESA participants $(N=5370)$. Age-adjusted $P$ values for trend across quartiles are shown at the highest quartile. 


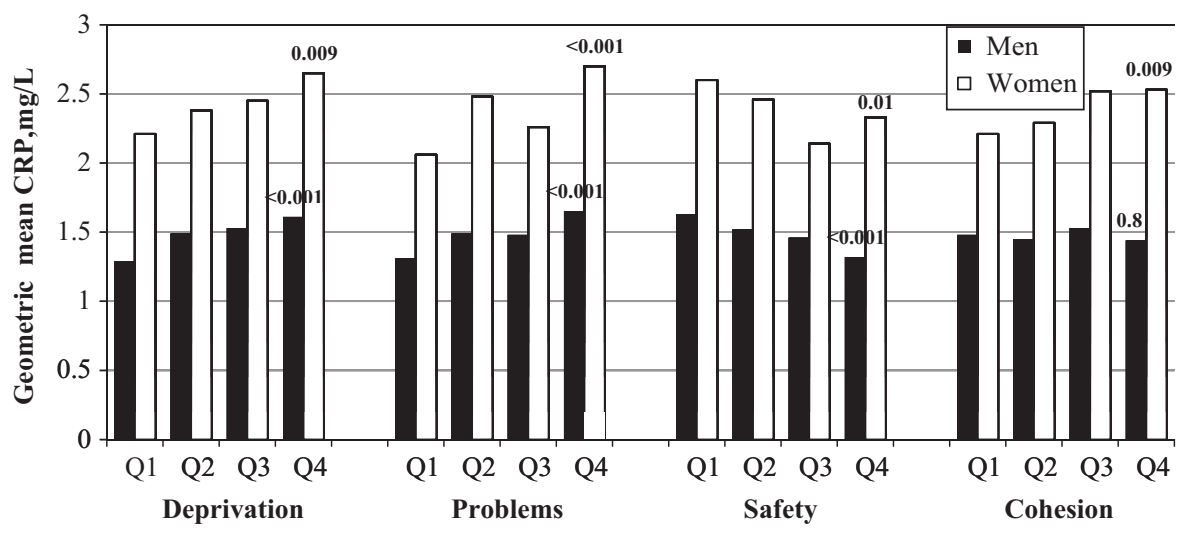

Lowest to highest quartile of neighborhood characteristic

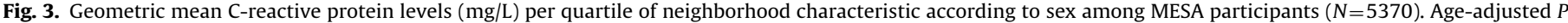
values for trend across quartiles are shown at the highest quartile.

Table 2

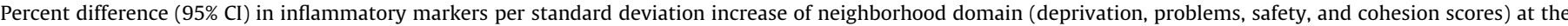
MESA baseline exam.

\begin{tabular}{|c|c|c|c|c|c|c|c|c|c|c|}
\hline & \multicolumn{2}{|l|}{ Model $1^{\mathrm{a}}$} & \multicolumn{2}{|l|}{ Model $2^{\mathrm{b}}$} & \multicolumn{2}{|l|}{ Model $3^{c}$} & \multicolumn{2}{|l|}{ Model $4^{\mathrm{d}}$} & \multicolumn{2}{|l|}{ Model $5^{\mathrm{e}}$} \\
\hline & $\begin{array}{l}\text { Percent } \\
\text { difference }\end{array}$ & $95 \% \mathrm{CI}$ & $\begin{array}{l}\text { Percent } \\
\text { difference }\end{array}$ & $95 \% \mathrm{CI}$ & $\begin{array}{l}\text { Percent } \\
\text { difference }\end{array}$ & $95 \% \mathrm{CI}$ & $\begin{array}{l}\text { Percent } \\
\text { difference }\end{array}$ & $95 \% \mathrm{CI}$ & $\begin{array}{l}\text { Percent } \\
\text { difference }\end{array}$ & $95 \% \mathrm{CI}$ \\
\hline \multicolumn{11}{|l|}{ Fibrinogen } \\
\hline Deprivation & 2.7 & $2.1,3.3$ & 2.1 & $1.5,2.7$ & 1.3 & $0.7,1.9$ & 0.9 & $0.3,1.5$ & 0.7 & $0.1,1.3$ \\
\hline Problems & 2.0 & $1.4,2.5$ & 1.7 & $1.2,2.3$ & 1.1 & $0.5,1.7$ & 0.9 & $0.3,1.5$ & 0.7 & $0.2,1.3$ \\
\hline Safety & -2.2 & $-2.8,-1.6$ & -1.8 & $-2.4,-1.3$ & -1.1 & $-1.7,-0.6$ & -0.9 & $-1.5,-0.4$ & -0.7 & $-1.3,-0.1$ \\
\hline Cohesion & -0.9 & $-1.4,-0.3$ & -0.6 & $-1.2,0.0$ & -0.6 & $-1.1,0.0$ & -0.5 & $-1.0,0.1$ & -0.4 & $-1.0,0.0$ \\
\hline \multicolumn{11}{|l|}{ IL-6 } \\
\hline Deprivation & 6.8 & $4.8,8.8$ & 4.6 & $2.5,6.7$ & 2.2 & $0.3,4.2$ & 0.5 & $-1.5,2.5$ & 0.3 & $-1.5,2.2$ \\
\hline Problems & 5.5 & $3.5,7.5$ & 4.8 & $2.8,6.8$ & 1.3 & $-0.6,3.2$ & 0.6 & $-1.3,2.5$ & -0.4 & $-2.2,1.4$ \\
\hline Safety & -6.6 & $-8.5,-4.8$ & -5.4 & $7.2,-3.4$ & -3.2 & $-5.0,-1.3$ & -2.2 & $-4.0,-0.3$ & -1.5 & $-3.3,0.3$ \\
\hline Cohesion & -2.1 & $-4.0,-0.2$ & -1.3 & $-3.2,0.6$ & -1.7 & $-3.5,0.1$ & -1.4 & $-3.2,0.4$ & -1.5 & $-3.2,0.2$ \\
\hline \multicolumn{11}{|l|}{ CRP } \\
\hline Deprivation & 9.0 & $5.3,12.8$ & 5.5 & $1.7,9.4$ & -0.8 & $-4.0,2.5$ & -3.6 & $-6.9,-0.3$ & -3.0 & $-6.2,0.2$ \\
\hline Problems & 9.8 & $6.1,13.6$ & 8.6 & $4.9,12.4$ & 0.4 & $-2.7,3.7$ & -0.6 & $-3.8,2.6$ & -2.0 & $-5.0,1.1$ \\
\hline Safety & -8.2 & $-11.5,-4.8$ & -6.4 & $-9.8,-2.9$ & -0.5 & $-3.7,2.8$ & 1.1 & $-2.2,4.5$ & 1.9 & $-1.2,4.9$ \\
\hline Cohesion & -0.8 & $-4.1,2.7$ & 0.3 & $-3.1,3.8$ & 0.0 & $-3.1,3.2$ & 0.4 & $-2.7,3.6$ & -0.2 & $-3.1,2.8$ \\
\hline
\end{tabular}

Using mixed linear regression models with random intercept at the census tract level.

a Model 1: adjusted for age and sex.

${ }^{b}$ Model 2: adjusted for age, sex, family income, and education.

c Model 3: adjusted for age, sex, and race/ethnicity.

${ }^{\mathrm{d}}$ Model 4: adjusted for age, sex, family income, education, and race/ethnicity.

e Model 5: adjusted for age, sex, family income, education, race/ethnicity, BMI, waist circumference, physical activity, dietary pattern score, smoking, alcohol intake, recent acute infection, medication use, impaired glucose tolerance, and diabetes.

interactions tested only two were statistically significant and no consistent sex differences were observed). There was also no clear evidence that associations of neighborhood characteristics with inflammatory markers differed by race/ethnicity (of 24 interactions tested only three were statistically significant and no consistent patterns emerged). Therefore, all subsequent analyses are pooled across the full sample and adjusted for sex and race/ ethnicity.

Table 2 shows the percent difference (95\% CI) in each inflammatory marker per standard deviation (SD) increase of neighborhood deprivation, problems, safety, and cohesion scores in the sample. In age-adjusted models (model 1), inflammatory markers were positively associated with neighborhood deprivation and problems scores, and inversely associated with neighborhood safety. Weaker and less consistent associations were observed for social cohesion (which was not associated with
CRP). Percent differences in inflammatory markers associated with a standard deviation difference in neighborhood deprivation, problems, or safety ranged from $2 \%$ to $10 \%$, depending on the neighborhood characteristic and the inflammatory marker.

Additional adjustment for income and education (model 2) slightly reduced the associations of deprivation, problems, and safety with inflammatory markers, with all estimates remaining statistically significant. In contrast, adjustment for race/ethnicity (model 3) substantially reduced all associations (even changing the direction of some of the associations for CRP). However, fibrinogen remained significantly associated with all neighborhood characteristics, and IL-6 remained significantly associated with deprivation and safety after adjustment for race/ethnicity. In models adjusted for both race/ethnicity and SES (model 4), neighborhood deprivation and neighborhood problems remained positively associated with fibrinogen and neighborhood safety 


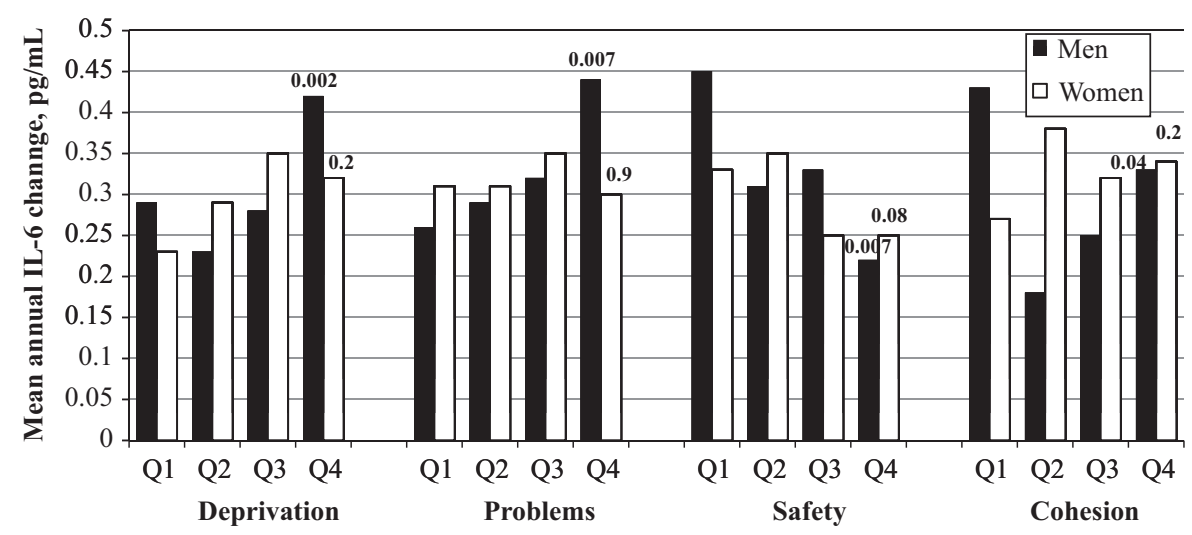

Lowest to highest quartile of neighborhood characteristic

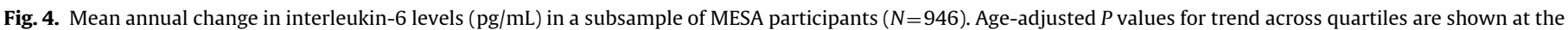
highest quartile.

remained inversely associated with fibrinogen and IL-6. An unexpected inverse association of deprivation with CRP was observed in the age, sex, race/ethnicity, and SES adjusted model.

Additional adjustment for BMI, waist circumference, physical activity, dietary score pattern, smoking, alcohol intake, recent acute infection, medication use, diabetes, and impaired fasting glucose (model 5 ) did not substantially modify associations with fibrinogen and weakened associations with IL-6, although all associations remained statistically significant or marginally statistically significant. No positive associations with CRP were observed in any of the race-adjusted models and results were similar when CRP was dichotomized at $3 \mathrm{mg} / \mathrm{L}$. In fully adjusted models (model 5) intraclass correlation coefficients for neighborhoods ranged from $0 \%$ to $1.3 \%$ and none were statistically significant. Associations of fibrinogen with neighborhood deprivation in age, sex, race/ethnicity, and SES adjusted models were reduced by $44 \%$ and were no longer statistically significant when neighborhood safety and problems were added to the models (percent difference associated with deprivation after adjustment for other neighborhood variables $0.595 \% \mathrm{CL}-0.3$ to 1.3 ).

Demographic characteristics of the longitudinal subsample ( $n=946)$ were generally similar to the full sample except that individuals in the subsample tended to have lower income and education and Chinese were not included in the follow-up subsample. The correlation between baseline and follow-up IL-6 measures was 0.60 . The mean (SD) annual change in IL-6 was $0.32 \mathrm{pg} / \mathrm{mL}$ (0.46). Change was positively associated with age, black or Hispanic race/ethnicity, obesity and smoking, and inversely associated with income and education (data not shown). Fig. 4 shows mean annual change in IL- 6 by quartiles of neighborhood characteristics. In general, higher baseline levels of neighborhood deprivation and problems, and lower levels of safety were associated with greater age-adjusted annual increases but no consistent associations were observed for social cohesion. There were no statistically significant interactions between neighborhood variables and sex.

Table 3 presents adjusted mean difference in annual change (95\% CI) in IL-6 levels per standard deviation increase of each neighborhood characteristic. Neighborhood deprivation and problems were positively associated with IL-6 change, whereas neighborhood safety was inversely associated with change. Mean differences in annual change per SD difference in neighborhood characteristics ranged from 0.03 to $0.06 \mathrm{pg} / \mathrm{mL}$ and were largely unchanged by level of adjustment. Adjusting for behavioral variables, acute infection, and diabetes at baseline reduced point estimates only slightly. Further adjustment for the same variables at follow-up study had no impact. Neighborhood cohesion was not significantly associated with IL-6 change in any model. Models that accounted for measurement error in baseline IL- 6 measures yielded similar results. Associations of IL-6 change with neighborhood deprivation in age, sex, race/ethnicity, and SES adjusted models were reduced by $17 \%$ when other neighborhood variables were included in models and remained statistically significant (mean difference in annual change associated with deprivation after adjustment for other neighborhood variables 0.05 95\% CI 0.01-0.09).

Approximately $14 \%$ of participants in the subsample moved residences from the baseline to the follow-up exams. Correlation of neighborhood deprivation score for original and new neighborhood for the individuals who moved was 0.57 . When analyses were restricted to those who did not move, all results presented here remained unchanged.

\section{Discussion}

In this large, multi-ethnic sample of community-dwelling adults, neighborhood characteristics were associated with levels of inflammatory markers. All four neighborhood domains investigated (deprivation, problems, safety, and cohesion) were crosssectionally associated with levels of fibrinogen, IL-6, and CRP after adjustment for age and sex. Fibrinogen remained significantly associated with all neighborhood characteristics except cohesion and IL-6 remained associated with safety after adjustment for race/ethnicity and socioeconomic indicators. These findings were not substantially modified by further risk factor adjustment. No positive associations with CRP were observed in any of the raceadjusted models. In longitudinal analyses, greater levels of neighborhood deprivation and problems, and lower levels of safety were significantly associated with greater increases in IL-6 over a 3-4 year period after adjustment for age, sex, race/ ethnicity, and socioeconomic factors. Point estimates were only slightly altered but confidence intervals became wider after additional risk factor adjustment so that only associations with deprivation remained statistically significant.

Few studies have investigated association of neighborhood characteristics with circulating levels of inflammatory markers. Petersen et al. (2008) found significant inverse associations between census tract SES indicators and IL-6 in a sample of 851 adults after adjusting for individual-level SES, race, and lifestyle factors (Petersen et al., 2008). Pollitt et al. (2007) found associations of census tracts SES with CRP and fibrinogen among 
Table 3

Mean difference in annual change (95\% CI) in IL-6 levels $(\mathrm{pg} / \mathrm{mL})$ per standard deviation increase of neighborhood domain in a subsample of MESA participants.

\begin{tabular}{|c|c|c|c|c|c|c|c|c|c|c|}
\hline \multirow{2}{*}{$\begin{array}{l}\text { Neighborhood } \\
\text { characteristic }\end{array}$} & \multicolumn{2}{|l|}{ Model $1^{\mathrm{a}}$} & \multicolumn{2}{|l|}{ Model $2^{b}$} & \multicolumn{2}{|l|}{ Model $3^{c}$} & \multicolumn{2}{|l|}{ Model $4^{\mathrm{d}}$} & \multicolumn{2}{|l|}{ Model $5^{\mathrm{e}}$} \\
\hline & Difference & $95 \% \mathrm{CI}$ & Difference & $95 \% \mathrm{CI}$ & Difference & $95 \% \mathrm{CI}$ & Difference & $95 \% \mathrm{CI}$ & Difference & $95 \% \mathrm{CI}$ \\
\hline Deprivation score & 0.06 & $0.02,0.09$ & 0.05 & $0.02,0.08$ & 0.05 & $0.02,0.09$ & 0.06 & $0.02,0.09$ & 0.05 & $0.02,0.09$ \\
\hline Problems score & 0.03 & $0.00,0.06$ & 0.03 & $0.00,0.06$ & 0.04 & $0.00,0.07$ & 0.04 & $0.00,0.07$ & 0.02 & $-0.01,0.05$ \\
\hline Safety score & -0.05 & $-0.09,-0.02$ & -0.04 & $-0.08,-0.01$ & -0.05 & $-0.08,-0.01$ & -0.04 & $-0.07,-0.01$ & -0.03 & $-0.07,0.00$ \\
\hline Cohesion score & -0.01 & $-0.04,0.03$ & 0.00 & $-0.04,0.03$ & -0.01 & $-0.04,0.02$ & -0.01 & $-0.04,0.02$ & 0.00 & $-0.02,0.03$ \\
\hline
\end{tabular}

Using mixed linear regression models with random intercept at the census tract level; mean follow-up time (range): 3.89 years (1.95-6.20).

a Model 1: adjusted for age and sex.

${ }^{\mathrm{b}}$ Model 2: adjusted for age, sex, family income, and education.

c Model 3: adjusted for age, sex, and race/ethnicity.

d Model 4: adjusted for age, sex, family income, education, and race/ethnicity.

e Model 5: adjusted for age, sex, family income, education, race/ethnicity, and at baseline: BMI, waist circumference, physical activity, dietary pattern score, smoking, alcohol intake, recent acute infection, medication use, impaired glucose tolerance, and diabetes.

white participants of the ARIC cohort after adjusting for individual-level SES but associations were less consistent and not statistically significant in African Americans (Pollitt et al., 2007). Only associations with fibrinogen remained statistically significant after risk factor adjustment. Consistent with this work, we found that living in neighborhoods with more deprivation was cross-sectionally associated with greater levels of fibrinogen after adjustment for race/ethnicity and SES. Addition of other risk factors for inflammation did not substantially modify the associations.

No other analyses of which we are aware have moved beyond neighborhood socioeconomic characteristics to investigate the specific neighborhood domains that may be relevant to inflammation. We found that greater levels of neighborhood problems were cross-sectionally associated with higher levels of fibrinogen, and lower levels of neighborhood safety were cross-sectionally associated with higher levels of fibrinogen and IL-6 after adjustment for race/ethnicity and individual-level SES. These associations were equivalent to the effect of approximately a $0.3 \mathrm{~kg} / \mathrm{m}^{2}$ difference in BMI in fully adjusted models.

The processes mediating these associations remain to be determined and could involve behavioral and/or psychosocial processes known to be linked to inflammation. There is abundant evidence linking behavioral factors to levels of fibrinogen and showing changes in fibrinogen when behaviors change (Abramson and Vaccarino, 2002; Bazzano et al., 2003; Balagopal et al., 2005; Chrysohoou et al., 2004; Nettleton et al., 2006). Psychosocial stress has also been linked to relatively short term changes in coagulation factors (Von Kanel et al., 2005, 2001). The cross-sectional analyses do not allow determination of the lag for these effects in our case but it is likely that contemporaneous conditions are proxying long term exposures given prior evidence of the residential stability of this cohort (Diez Roux et al., 2008). The fact that associations persisted after adjustment for established behavioral risk factors provides some albeit limited evidence that behavioral factors do not fully explain these associations.

Neighborhood social cohesion was not consistently associated with any inflammatory markers. This may be due to limitations in the measure of social cohesion used. Alternatively, social cohesion may serve to buffer the effects of stress without directly affecting levels of inflammation (Stockdale et al., 2007). Associations of deprivation with fibrinogen were reduced by nearly a half when neighborhood safety and problems were accounted for, suggesting that these factors (and their psychosocial or behavioral consequences) may in part explain why deprived neighborhoods are associated with higher fibrinogen levels.

Our analyses are among the first to examine associations of neighborhood characteristics with changes in inflammatory markers over time. Greater levels of neighborhood deprivation and problems, and lower levels of safety were significantly associated with greater increases in IL- 6 over time after adjustment for race and socioeconomic factors. Risk factor adjustment did not substantially modify these associations. Mean differences in annual changes associated with an SD difference in the neighborhood predictors were substantial and in the order of $22-26 \%$ of the mean annual change $(0.32 \mathrm{pg} / \mathrm{mL})$ for neighborhood deprivation, $13-17 \%$ for problems, and $17-26 \%$ for safety. This suggests that neighborhood conditions may significantly impact longitudinal changes in IL-6 associated with aging. However in contrast to cross-sectional analyses, neighborhood problems and safety explained $<20 \%$ of the differences in longitudinal change associated with deprivation.

We investigated three commonly studied inflammatory markers previously linked to cardiovascular events. The three markers are physiologically linked. Interleukin 6 (IL-6) is pro-inflammatory cytokine, which stimulates the production of acute phase reactants like CRP and fibrinogen. In addition, fibrinogen is also a key coagulation factor and increases clotting activity (Tracy, 1999). Prior work on neighborhoods and inflammation has investigated different markers but results have not always been consistent. Petersen et al. (2008) found associations of neighborhood SES with both CRP and IL-6 after adjustment for race and individual-level SES but only associations with IL-6 remained statistically significant after adjustment for lifestyle factors. Pollitt et al. (2007) found stronger associations of neighborhood SES with CRP than with fibrinogen in white ARIC participants, but only associations with fibrinogen remained statistically significant after risk factor adjustment. In studies of individual-level SES, at least two studies have reported more consistent associations with fibrinogen than with CRP (Pollitt et al., 2008; Thomas et al., 2005) but others have reported similar associations for CRP and fibrinogen (Jousilahti et al., 2003; Ramsay et al., 2008). In prior MESA analyses, individual-level SES was similarly related to CRP and IL-6 (Ranjit et al., 2007a).

We have no compelling physiologic explanation for why fibrinogen was the marker most consistently associated with neighborhood characteristics in cross-sectional analyses. It is notable that of the three inflammatory markers we examined it is the only one that also plays an important role in coagulation processes, which have been consistently linked to social exposures in prior work (Davis et al., 1995; Markowe et al., 1985; Rosengren et al., 1990; Tsutsumi et al., 1999). Differential measurement properties of the inflammatory markers could play a role. Of note, we did document associations of neighborhood characteristics with within-person changes in IL-6 over time. Because of its role as a pro-inflammatory cytokine, it has been 
postulated that IL6 may play a key role in behavioral, stress, and inflammatory processes linked to cardiovascular disease (Yudkin et al., 2000). Unfortunately, the absence of longitudinal data on CRP and fibrinogen made it impossible to contrast longitudinal associations with the three markers.

A potential explanation for the differences in results across inflammatory markers is intractable confounding by race/ethnicity. Race/ethnicity was a much stronger predictor of IL-6 and CRP than that of fibrinogen in our data. Associations of race/ethnicity with CRP were especially strong. We have no clear explanation for why this is the case, but the fact that neighborhood characteristics are also strongly associated with race made it difficult to isolate any associations of neighborhood characteristics with CRP in our data when race/ethnicity was controlled. In contrast, fibrinogen was not as strongly associated with race/ethnicity. Longitudinal changes in IL-6 levels were not as strongly confounded by race/ ethnicity or SES, possibly contributing to our ability to detect associations of neighborhood factors with within person changes in IL-6 after adjustment for race and SES.

The MESA cohort was free of clinical CVD at recruitment. The inclusion of a healthy sample could have resulted in the selective exclusion of persons with high levels of inflammatory markers from disadvantaged neighborhoods leading us to underestimate true effects. Census tracts are undoubtedly imperfect proxies for neighborhoods, or more generally for the spatial scale potentially relevant to inflammation although they have been shown to be reasonable proxies in prior work (Clapp and Wang, 2006; Krieger et al., 2002; Mujahid et al., 2007). Measurement error in assessing neighborhood attributes is also a possibility. We aggregated responses across multiple respondents to minimize error associated with individual subjectivity but there is no doubt that important misspecification of the neighborhood level variables remains, possibly limiting our ability to detect associations with distant downstream factors like inflammation. We attempted to investigate the extent to which associations were explained by established risk factors for inflammation (such as BMI and behaviors) but measurement error in these factors limits our ability to draw firm conclusions regarding "independent effects" of neighborhood contexts.

Important strengths of this study are the population-based sample and the ability to examine whether neighborhood factors are associated with longitudinal changes in inflammatory markers over time. In sum, we found that neighborhood deprivation, problems, and safety were significantly associated with circulating fibrinogen levels in cross-sectional analyses. In addition, adverse neighborhood environments were consistently associated with greater increases in IL-6 over time after adjusting for race/ ethnicity and socioeconomic indicators. The longitudinal results suggest that the role of inflammatory processes in mediating neighborhood differences in cardiovascular risk deserved further exploration.

\section{Competing interests}

None declared.

\section{Acknowledgements}

This work was supported in part by R01 HL76831 and by the Michigan Center for Integrative Approaches to Health Disparities P60 MD002249. MESA was supported by Contracts N01-HC95159 through N01-HC-95165 and N01-HC-95169 from the National Heart, Lung, and Blood Institute. The authors thank the other investigators, the staff, and the participants of the MESA study for their valuable contributions. A full list of participating MESA investigators and institutions can be found at http://www. mesa-nhlbi.org. NHLBI representatives review MESA manuscript proposals prior to submission and participate in MESA Steering Committee meetings.

\section{References}

Abramson, J.L., Vaccarino, V., 2002. Relationship between physical activity and inflammation among apparently healthy middle-aged and older US adults. Arch. Intern. Med. 162, 1286-1292.

Acevedo-Garcia, D. 2000. Residential segregation and the epidemiology of infectious diseases. Soc. Sci. Med. 51, 1143-1161.

Ainsworth, B.E., Irwin, M.L., Addy, C.L., Whitt, M.C., Stolarczyk, L.M., 1999. Moderate physical activity patterns of minority women: the cross-cultural activity participation study. J. Womens Health Gender Based Med. 8, 805-813.

Auchincloss, A.H., Diez Roux, A.V., Brown, D.G., Erdmann, C.A., Bertoni, A.G., 2008. Neighborhood resources for physical activity and healthy foods and their association with insulin resistance. Epidemiology 19, 146-157.

Balagopal, P., George, D., Patton, N., Yarandi, H., Roberts, W.L., Bayne, E., Gidding, S., 2005. Lifestyle-only intervention attenuates the inflammatory state associated with obesity: a randomized controlled study in adolescents. J. Pediatr. 146, 342-348.

Bazzano, L.A., HE, J., Muntner, P., Vupputuri, S., Whelton, P.K., 2003. Relationship between cigarette smoking and novel risk factors for cardiovascular disease in the United States. Ann. Intern. Med. 138, 891-897.

Bild, D.E., Bluemke, D.A., Burke, G.L., Detrano, R., Diez Roux, A.V., Folsom, A.R., Greenland, P., Jacobsjr, D.R., Kronmal, R., Liu, K., Nelson, J.C., O’leary, D., Saad, M.F., Shea, S., Szklo, M., Tracy, R.P., 2002. Multi-ethnic study of atherosclerosis: objectives and design. Am. J. Epidemiol. 156, 871-881.

Chaix, B., Rosvall, M., Merlo, J., 2007. Neighborhood socioeconomic deprivation and residential instability: effects on incidence of ischemic heart disease and survival after myocardial infarction. Epidemiology 18, 104-111.

Chrysohoou, C., Panagiotakos, D., Pitsavos, C., Das, U., Stefanadis, C., 2004. Adherence to the Mediterranean diet attenuates inflammation and coagulation process in healthy adults: the ATTICA study. J. Am. Coll. Cardiol. 44, 152-158.

Clapp, J.M., Wang, Y., 2006. Defining neighborhood boundaries: are census tracts obsolete? J. Urban Econ. 59 259-284.

Cubbin, C., Winkleby, M.A., 2005. Protective and harmful effects of neighborhoodlevel deprivation on individual-level health knowledge, behavior changes, and risk of coronary heart disease. Am. J. Epidemiol. 162, 559-568.

Davis, M.C., Matthews, K.A., Meilahn, E.N., Kiss, J.E., 1995. Are job characteristics related to fibrinogen levels in middle-aged women? Health Psychol. 14 310-318.

Diez-Roux, A.V., Nieto, F.J., Tyroler, H.A., Crum, L.D., Szklo, M., 1995. Social inequalities and atherosclerosis: the atherosclerosis risk in communities study. Am. J. Epidemiol. 141, 960-972.

Diez Roux, A.V., Auchincloss, A.H., Astor, B.C., Barr, R.G., Greene Franklin, T. Kaufman, J.D., Keeler, J., Raghunathan, T.E., 2008. Long-term exposure to ambient particulate matter and prevalence of subclinical atherosclerosis in the multiethnic study of atherosclerosis (MESA). Am. J. Epidemiol. 167, 667-675.

Diez Roux, A.V., Mair, C., 2010. Neighborhoods and health. Ann. N. Y. Acad. Sci. $1186,125-145$.

Diez Roux, A.V., Merkin, S.S., Arnett, D., Chambless, L., Massing, M., Nieto, F.J., Sorlie, P., Szklo, M., Tyroler, H.A., Watson, R.L., 2001. Neighborhood of residence and incidence of coronary heart disease. N. Engl. J. Med. 345, 99-106.

Echeverria, S., Diez-Roux, A., LINK, B., 2004. Reliability of self-reported neighborhood characteristics. J. Urban Health 81, 682-701.

Echeverría, S., Diez-Roux, A.V., Shea, S., Borrell, L.N., Jackson, S., 2008. Associations of neighborhood problems and neighborhood social cohesion with mental health and health behaviors: the multi-ethnic study of atherosclerosis. Health Place $14,853-865$.

Franzini, L., Spears, W., 2003. Contributions of social context to inequalities in years of life lost to heart disease in Texas, USA. Soc. Sci. Med. 57, 1847-1861.

Hardin, J., Schmiediche, H., Carroll, R., 2003. The regression-calibration method for fitting generalized linear models with additive measurement error. Stata J. 3 , 361-372.

Jousilahti, P., Salomaa, V., Rasi, V., Vahtera, E., Palosuo, T., 2003. Association of markers of systemic inflammation, $C$ reactive protein, serum amyloid $A$, and fibrinogen, with socioeconomic status. J. Epidemiol. Community Health 57, 730-733.

Krieger, N., Waterman, P., Chen, J.T., Soobader, M.-J., Subramanian, S.V., Carson, R., 2002. Zip code caveat: bias due to spatiotemporal mismatches between zip codes and US census-defined geographic areas - the public health disparities geocoding project. Am. J. Public Health 92, 1100-1102.

Leclere, F.B., Rogers, R.G., Peters, K., 1998. Neighborhood social context and racial differences in women's heart disease Mortality. J. Health Soc. Behav. 39, 91-107.

Markowe, H.L., Marmot, M.G., Shipley, M.J., Bulpitt, C.J., Meade, T.W., Stirling, Y., Vickers, M.V., Semmence, A., 1985. Fibrinogen: a possible link between social class and coronary heart disease. Br. Med. J. (Clin. Res. Ed.) 291, 1312-1314.

Mcdade, T.W., Hawkley, L.C., Cacioppo, J.T., 2006. Psychosocial and behavioral predictors of inflammation in middle-aged and older adults: the Chicago health, aging, and social relations study. Psychosom. Med. 68, 376-381. 
Mujahid, M.S., Diez Roux, A.V., Morenoff, J.D., Raghunathan, T., 2007. Assessing the measurement properties of neighborhood scales: from psychometrics to ecometrics. Am. J. Epidemiol. 165, 858-867.

Nazmi, A., Victora, C.G., 2007. Socioeconomic and racial/ethnic differentials of Creactive protein levels: a systematic review of population-based studies. BMC Public Health 7, 212.

Nettleton, J., Steffen, L., Mayer-Davis, E., Jenny, N., Jiang, R., Herrington, D., Jacobs, D.J., 2006. Dietary patterns are associated with biochemical markers of inflammation and endothelial activation in the multi-ethnic study of atherosclerosis (MESA). Am. J. Clin. Nutr. 83, 1369-1379.

O'neill, M.S., Jerrett, M., Kawachi, I., Levy, J.I., Cohen, A.J., Gouveia, N., Wilkinson, P., Fletcher, T., Cifuentes, L., Schwartz, J., 2003. Health, wealth, and air pollution: advancing theory and methods. Environ. Health Perspect. 111, 1861-1870.

Pahor, M., Elam, M.B., Garrison, R.J., Kritchevsky, S.B., Applegate, W.B., 1999. Emerging noninvasive biochemical measures to predict cardiovascular risk. Arch. Intern. Med. 159, 237-245.

Petersen, K.L., Marsland, A.L., Flory, J., Votruba-Drzal, E., Muldoon, M.F., Manuck, S.B., 2008. Community socioeconomic status is associated with circulating interleukin-6 and C-reactive protein. Psychosom. Med. 70, 646-652.

Pollitt, R.A., Kaufman, J.S., Rose, K.M., Diez-Roux, A.V., Zeng, D., Heiss, G., 2007. Early-life and adult socioeconomic status and inflammatory risk markers in adulthood. Eur. J. Epidemiol. 22, 55-66.

Pollitt, R.A., Kaufman, J.S., Rose, K.M., Diez-Roux, A.V., Zeng, D., Heiss, G., 2008. Cumulative life course and adult socioeconomic status and markers of inflammation in adulthood. J. Epidemiol. Community Health 62, 484-491.

Ramsay, S., Lowe, G.D.O., Whincup, P.H., Rumley, A., Morris, R.W., Wannamethee, S.G., 2008. Relationships of inflammatory and haemostatic markers with social class: results from a population-based study of older men. Atherosclerosis 197, 654-661.

Ranjit, N., Diez-Roux, A.V., Sanchez, B., Seeman, T., Shea, S., Shrager, S., Watson, K., 2009b. Association of salivary cortisol circadian pattern with cynical hostility: multi-ethnic study of atherosclerosis. Psychosom. Med. doi:10.1097/PSY.0b013e3181ad23e7.

Ranjit, N., Diez-Roux, A.V., SHEA, S., Cushman, M., Ni, H., Seeman, T., 2007a. Socioeconomic position, race/ethnicity, and inflammation in the multi-ethnic study of atherosclerosis. Circulation 116, 2383-2390.

Ranjit, N., Diez-Roux, A.V., Shea, S., Cushman, M., Seeman, T., Jackson, S.A., Ni, H. 2007b. Psychosocial factors and inflammation in the multi-ethnic study of atherosclerosis. Arch. Intern. Med. 167, 174-181.
Rosengren, A., Wilhelmsen, L., Welin, L., Tsipogianni, A., Teger-Nilsson, A.C., Wedel H., 1990. Social influences and cardiovascular risk factors as determinants of plasma fibrinogen concentration in a general population sample of middle aged men. Br. Med. J. 300, 634-638.

Stockdale, S.E., Wells, K.B., Tang, L., Belin, T.R., Zhang, L., Sherbourne, C.D., 2007. The importance of social context: neighborhood stressors, stress-buffering mechanisms, and alcohol, drug, and mental health disorders. Soc. Sci. Med. 65, 1867-1881.

Sundquist, K., Winkleby, M., Ahlen, H., Johansson, S.-E., 2004. Neighborhood socioeconomic environment and incidence of coronary heart disease: a follow-up study of 25,319 women and men in Sweden. Am. J. Epidemiol. 159, 655-662.

Thomas, N.E., Cooper, S.M., Williams, S.R., Baker, J.S., Davies, B., 2005. Fibrinogen, homocyst(e)ine, and $\mathrm{C}$-reactive protein concentrations relative to sex and socioeconomic status in British young people. Am. J. Hum. Biol. 17, 809-813.

Tracy, R.P., 1999. Inflammation markers and coronary heart disease. Curr. Opin. Lipidol. 10, 435-441.

Tsutsumi, A., Theorell, T., Hallqvist, J., Reuterwall, C., DE Faire, U., 1999. Association between job characteristics and plasma fibrinogen in a normal working population: a cross sectional analysis in referents of the SHEEP study. Stockholm heart epidemiology program. J. Epidemiol. Community Health 53, 348-354.

Von Kanel, R., Kudielka, B.M., Hanebuth, D., Preckel, D., Fischer, J.E., 2005. Different contribution of interleukin-6 and cortisol activity to total plasma fibrin concentration and to acute mental stress-induced fibrin formation. Clin. Sci. 109, 61-67.

Von Kanel, R., Mills, P.J., Fainman, C., Dimsdale, J.E., 2001. Effects of psychologica stress and psychiatric disorders on blood coagulation and fibrinolysis: a biobehavioral pathway to coronary artery disease? Psychosom. Med. 63 531-544.

Willerson, J.T., Ridker, P.M., 2004. Inflammation as a cardiovascular risk factor Circulation 109 II-2-10.

Wilson, T.W., Kaplan, G.A., Kauhanen, J., Cohen, R.D., Wu, M., Salonen, R., Salonen, J.T., 1993. Association between plasma fibrinogen concentration and five socioeconomic indices in the kuopio ischemic heart disease risk factor study. Am. J. Epidemiol. 137, 292-300.

Winkleby, M., Sundquist, K., Cubbin, C., 2007. Inequities in CHD incidence and case fatality by neighborhood deprivation. Am. J. Prev. Med. 32, 97-106.

Yudkin, J.S., Kumari, M., Humphries, S.E., Mohamed-Ali, V., 2000. Inflammation, obesity, stress and coronary heart disease: is interleukin-6 the link? Atherosclerosis 148 209-214. 